$\xi=-1$

\title{
Traffic Flow Modeling on Road Networks Using Cellular Automata Theory
}

\author{
A. Chechina *, N. Churbanova, M. Trapeznikova, A. Ermakov, M. German \\ Keldysh Institute of Applied Mathematics RAS, 4 Miusskaya Square, Moscow, Russia \\ *Email: chechina.antonina@yandex.ru
}

\begin{abstract}
The paper deals with mathematical modeling of traffic flows on urban road networks. The original model is based on the cellular automata theory and presents a generalization of Nagel-Schreckenberg model to a multilane case.

Numerical realization of the model is represented in a form of the program package that consists of two modules: User Interface and Visualization module (for setting initial conditions and modelling parameters and visual representation of calculations) and Computation module (for calculations).

Computations are carried out for each element of the road (i.e. T or X type intersection, straight road fragment) separately and in parallel, that allows performing calculations on various complex road networks. Different kinds of average characteristics (e.g. the capacity of the crossroad) can be also obtained using the program package.
\end{abstract}

Keywords: Cellular automata; traffic flow modeling.

\section{Introduction}

\subsection{Cellular automata theory in traffic flow modeling}

Cellular automata theory in application to traffic flow modeling is a widely used approach which allows taking into consideration a lot of nuances of real traffic. Since Nagel and Schreckenberg first presented their model in 1992 [1], a lot of scientists used it to create traffic flow models (see, for example, [3]). This process still continues, because, as computational facilities develop, more precise and complicated models can be created and applied for computations on large-scale road networks.

Traffic flow models based on cellular automata theory are fully discrete. The road is divided into equal cells, each cell can either contain a vehicle, or be empty. The distance along and across the road is measured in the number of cells. Time steps are usually equal to 1 second, the speed of the vehicle is the number of cells the vehicle can overcome during one time step. Each time step the cell state update is made according to certain logical rules. This approach has both advantages and disadvantages: on the one hand, simple logical algorithms allow to take into consideration different characteristics of vehicular traffic, such as, for example, driving strategies; on the other hand - discrete speed and acceleration introduce certain limitations into a model. Also, the fixed size of the cell (usually 7.5 meters) makes it difficult to model heterogeneous traffic that includes long vehicles, such as trucks and buses. Nevertheless, it is possible to overcome these difficulties (to a certain point, at least) for the tasks where it is needed, that's why the approach remains rather popular.

\subsection{The original model}

As opposed to the classic Nagel-Schreckenberg model [1], the developed model is two-dimensional and allows to depict multilane traffic.

A cell is 7.5 meters long, the time step is 1 second. Each car has a set of parameters: unique ID, maximum speed, current speed and final destination.

A road fragment with cars is shown in Fig.1. The car color in the picture corresponds to different destination points (road exits, turns, etc.).

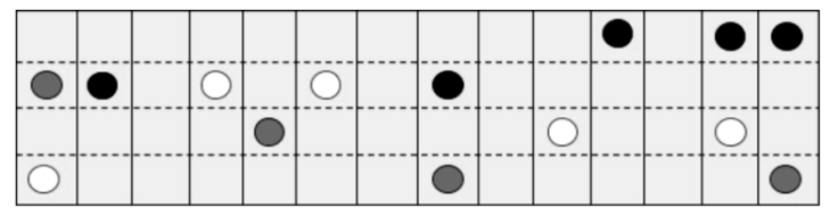

Fig. 1: Multilane road fragment with cars.

Each time step cell state update is carried out according to the following rules:

- a vehicle changes a lane if it is necessary (to reach the desired destination or to drive around an obstacle), it is advantageous for a driver (leads to speed increase and/or density decrease) and it is possible (i.e. if the lane change is allowed and the target cell is empty);

- a vehicle moves along the road according to the classic rules for the one-lane traffic [1].

\section{Algorithms included in the model}

\subsection{Changing lanes}

Lane changing takes place under following conditions:

- a vehicle is located in a domain where lane change is allowed; 
- $\quad$ lane change leads to the speed increase (density decrease) or is necessary to reach the destination;

- there is an obstacle not far away on the current lane;

- $\quad$ the target cell is empty;

- the safety condition is satisfied - on the target lane the distance behind is greater or equal to the maximal/current velocity of the previous car (cautious/aggressive drivers), the distance in front is greater or equal to the concerned vehicle velocity;

- $\quad$ lane change takes place with a given probability.

\subsection{The algorithm of crossroad overcoming}

- Within 100 meters before traffic lights the vehicle changes lane under its purpose according to the road laws.

- A vehicle accelerates or decelerates according to the Nagel-Schreckenberg model.

- Additional speed decreasing takes place under the following conditions:

if a vehicle is located near the turning point (at the turning point it stops);

if a traffic light is red;

- $\quad$ if there is the collision threat on the crossroad.

- A vehicle moves under the foregoing rules with randomization.

- A vehicle turns if it is located in the turning point and has got the corresponding target.

\subsection{Forming the entrance queue}

According to the entrance flow value for the given direction that is set by user before the calculation, time steps on which new vehicles should be added in the system are specified. At a given time step the vehicle appears in the entrance cell of the right lane if it is not occupied by another vehicle. If it happens to be occupied, other lanes are checked. If there is a traffic jam and all the entrance cells in the given direction contain vehicles already, the car can't enter and it is added into the queue. As soon as one of the entrance cells vacates, the car appears on the road.

\subsection{Road narrowing/widening}

Each driver is able to "see" if the road narrows down some distance ahead. In this case, the driver tries to change lane if his lane is going to disappear, even if the speed in the target lane is lower than the speed in his current lane and the traffic over there is denser. Some drivers in the target lane, if they are "cooperative" (see section 2.6), can stop to let the driver change his lane.

In case of the road widening, drivers react only when they reach it, following the general "lesser density/higher velocity" rule, i.e. seeing the empty lane, they move there for more comfortable driving.

\subsection{Driving around an obstacle}

If there is an obstacle in the middle of the road (for example, a broken car or cars after a road accident), a driver is able to "see" it before it reaches it, like in the case of a road narrowing. He also tries to drive around it, first determining which direction of lane changing is preferable, and then he changes lanes till he reaches a free one, with or without help of "cooperative" drivers.

\subsection{Different driving strategies}

Each driver has his own driving style: ones are more determined, change lanes more easily, others are more cautious, they wait for a larger gap to move to the neighboring lane. Their behavior influences the overall situation on the road. To reflect that, the following driver types were included in the model:
- "Cautious" drivers change lanes only if the gap between the target cell and the first occupied cell upstream is larger than the maximum speed;

- $\quad$ "Aggressive" drivers change lanes if the gap between the target cell and the first occupied cell upstream is larger than the actual speed of the vehicle which is situated in the first occupied cell;

- $\quad$ "Cooperative" drivers can be either cautious or aggressive. The percentage of cooperative drivers in the system can vary. These drivers:

- $\quad$ slow down $(\mathrm{v}=\mathrm{v}-1 ; \mathrm{v} \geq 1)$, if they see a traffic jam before an obstacle or a road bottleneck on the neighbouring lane;

if there is a jam before the obstacle on the neighbouring lane, and there are drivers that want to change their lane, cooperative drivers stop and let them pass; wait for several time steps if the car from the other lane can't move immediately, because the target cell is occupied by another car.

\section{Numerical implementation and tests}

Numerical realization of the model is represented in a form of the original program package created by the authors and called CAM2D - "Cellular Automata Model - 2-Dimensional". It consists of two modules: User Interface and Visualization module (for setting initial conditions and modelling parameters and calculations visual representation) and Computation module (for calculations).

The computation module is written on $\mathrm{C} / \mathrm{C}++$ and uses Glut library for the alternative visualisation means that are used to debug the program, and MPI library for parallel calculations. The Computation module has a module structure: separate sub-programmes simulate traffic on different types of road elements in parallel, with data exchange on the boundaries. Those road elements are: T- or X-type signalized intersections, on-ramp, straight road with and without traffic lights, straight road with a widening (Fig. 2). The smaller squares represent cars, the larger squares (at T-Cross and X-cross) - traffic lights. Cars have different colours according to planned destinations. Each road type can have any number of lanes.

Road accidents can be also simulated using CAM-2D (see Fig 2, right frame in the bottom row). Road accidents can not happen inside the model due to the safety rules: a car always slows down approaching an obstacle or another car in the lane, and it doesn't change its lane if the gap in the target lane is not enough to do it safely. But it is possible to model consequences of an accident that might be necessary for real-time online modeling of city traffic in order to develop the measures to avoid traffic jams and evaluate the efficiency of these measures. It is also possible to

evaluate how much the capacity of a road will decrease after the accident.

Having modules simulating various types of the roads, we can create numerous road networks, matching real road networks of the city or a district. Different kinds of problems can be solved using CAM-2D, for example, the capacity of the crossroad depending on the traffic lights regimes [2], or the influence of changes in road infrastructure on traffic situation.

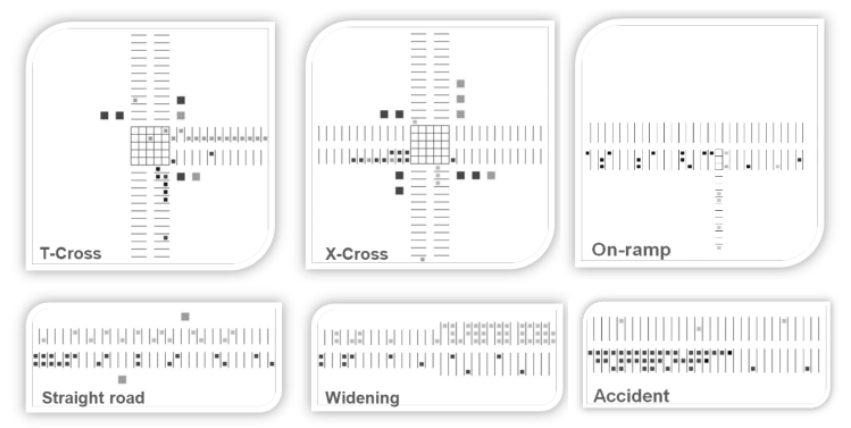


Fig. 2: Simulating traffic on various road fragments.

\section{Modeling traffic on road networks}

As it was mentioned before, the main problem to be solved using CAM-2D program package is to simulate traffic on road networks of different scale. In case of smaller networks, such as several neighboring intersections inside a city district, it makes sense to compute each intersection on a different processor, in order to use unified elements shown in Fig. 2, although, in this case the processor won't be fully loaded. In case of larger networks it is reasonable to use larger fragments for each processor, combining different intersections.

For visualizing traffic on networks the User Interface and Visualization module of CAM-2D is used. It allows seeing cars moving through a network consisting of any number of intersections of all kinds (Fig. 3).

Traffic flow simulation on the combination of intersections that correspond to the real road network in Moscow was carried out using CAM-2D (see Fig. 4, scheme on the left and the result of simulation using Visualization module on the right). These computations allow, using real data on the flow rates at different time periods, to determine what traffic lights settings would allow to maximize the capacity of this road section.

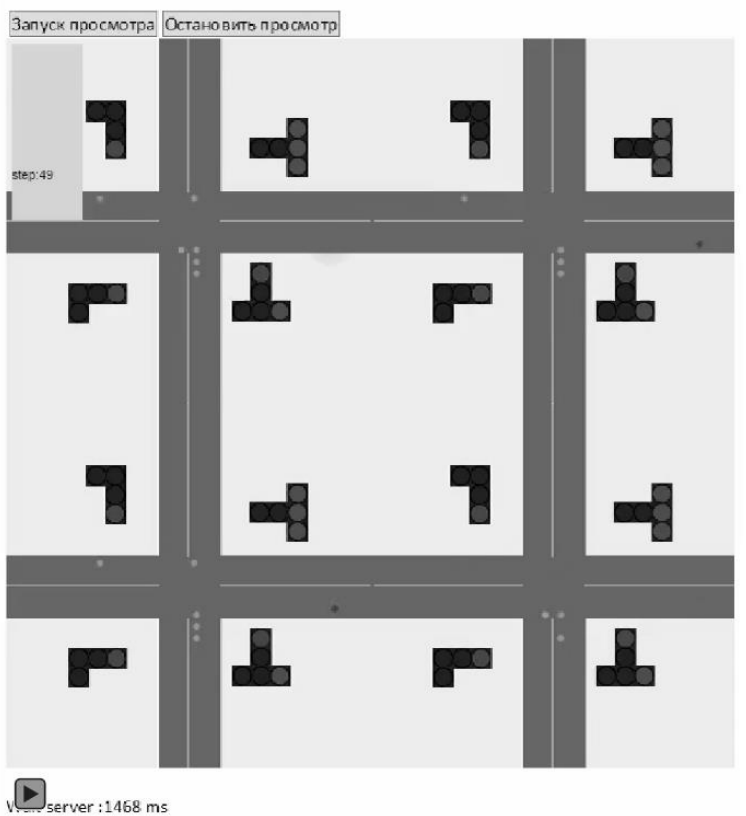

Fig. 3: Simulating traffic on a small road network.
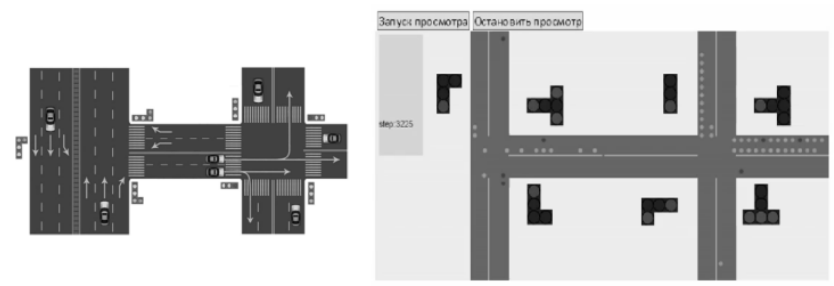

Fig. 4: Simulating traffic on two neighboring intersections.

\section{Conclusion}

The model and algorithms developed showed results comparable with the simulation by the standard program software. The created program package can be used for traffic modeling on city road networks, taking into consideration different driving strategies and evaluating the efficiency of measures taken in order to decrease traffic jams.
Due to the inner parallelism and the simplicity of the numerical algorithm the calculations can be carried out using high performance supercomputers on large-scale road networks in real time. One of the planned directions of further investigations is an adjustment of the model to match with the well-known three-phase theory by B. Kerner (see, for example, [4]).

\section{Acknowledgement}

The work is supported by RFBR projects No 16-01-00347, 18-0100405.

\section{References}

[1] K. Nagel, M. Schreckenberg, "A Cellular Automation Model for Freeway Traffic", J. Phys. I France, Vol.2 (1992), p. 2221

[2] A. Chechina, N. Churbanova, M. Trapeznikova, "Different Approaches to the Multilane Traffic Simulation", Traffic and Granular Flow '13, M. Chraibi et al. (Eds.), Springer, (2015) pp. 361-368

[3] S. Maerivoet, B. De Moor, "Cellular automata models of road traffic", Physics Reports Vol .419 (2005) pp. 1-64

[4] B. Kerner, Breakdown in Traffic Networks. Fundamentals of Transportation Science, Springer, Germany (2017) 652 p. 